\title{
LOW LUMINOSITY STARS AND WHITE DWARFS
}

\author{
W. J. LUYTEN
}

University of Minnesota, Minneapolis, Minn., U.S.A.

(1) When discussing properties of stars of low luminosity we should first define what we mean by that term. My own feeling is that a bolometric luminosity of 0.001 of that of the Sun appears to be a reasonable upper limit for 'low luminosity'. Further, it is obvious that, in order to find any numbers of them, one must go down to the very faintest objects, near the limit of what our present telescopes can show. This, in turn, means that virtually no accurate magnitudes, colors, or parallaxes will be available, and that our observational data must be obtained almost exclusively from proper motion surveys for very faint stars. Thus we end up by saying that only the 48-in. Palomar Schmidt telescope can produce large numbers of them. Such a statement is, of course, an oversimplification, but how much of one can be judged from the fact that when three years ago I published a catalogue of 1055 such stars only 36 had come from other sources, and 1019, or $96 \frac{1}{2} \%$ from the Palomar Schmidt plates. We have now processed more than 400 pairs of plates with our automated-computerized blink machine, and we have at least another 1000 new, low-luminosity stars, and the Palomar Schmidt contribution reaches at least $98 \%$.

In getting back to the data, accepting $L=0.001 \odot$ or $M_{\text {bol }}=12.2$ as our upper limit, and realizing that most of these stars will probably be very red, it means we have to look for stars with $M_{p g}$ larger than +16.5 . Since no parallaxes are known, we must finally identify these objects statistically from their proper motions. If we assume that, on the average, they have tangential velocities of $75 \mathrm{~km} \mathrm{~s}^{-1}$, this means that the quantity $H=m+5+5 \log \mu=M+5 \log T$ must be larger than $22.5 \mathrm{pg}$. At every step of this derivation large uncertainties enter - still, $I$ believe this is a workable way of finding low-luminosity stars. I have also tried to extend this same line of reasoning to stars less red, but there one almost certainly runs into white dwarfs and degenerates, and objects will be included in the list because of extraordinary large velocities up to almost ten times what I have assumed here, which, in turn, would mean stars with luminosities one hundred times greater than the limit assumed.

In order to reach a value of $H$ larger than $22.5 \mathrm{pg}$ a star must have the minimum proper motion as shown below, for various apparent photographic magnitudes. It is not surprising that no red star brighter than the fourteenth photographic magni-

$\begin{array}{ll}m_{p g} & \mu_{\mathrm{m} \mathrm{n}} \\ & - \\ 14 & 5 \\ 16 & 2 \\ 18 & 0.8 \\ 20 & 0.32 \\ 21.5 & 0.16\end{array}$


tude can make it - Barnard's star, Proxima Centauri and the components of L 726-8 do not make the grade.

When we turn to the statistical discussion of the properties of these stars we must remember that virtually all our information comes from proper motion surveys, hence we can expect that, selectively, our stars will be overwhelmingly high-velocity stars. If there are stars fainter than, say, $m=18 \mathrm{pg}$ (apparent), with small enough tangential velocities, and therefore small proper motions to belong to our group of low-luminosity stars, we have no means of discovering them at present.

The frequency in space of these low-luminosity stars is important because they provide the bulk of the material near, and below the maximum of the luminosity function. Ever since Kapteyn made his first determination of the Luminosity Function in 1918 and found the maximum to lie at $M=+7.7$ (vis) each subsequent determination pushed the maximum fainter. When I analyzed the Bruce data in 1937, I derived $M=+14.5 \mathrm{pg}$ but thirty years later when the first results from the Palomar Survey became available, I went down to $M=+15.7 \mathrm{pg}$. A few months ago La Bonte and I completed the Blink-machine analysis of a region of some $3000 \mathrm{sq}$ deg near the South Galactic Pole, down to apparent magnitude $21 \mathrm{pg}$, for motions larger than 0.18 annually. The first preliminary analysis indicates that the maximum lies at $M=+15.4$ - so for the first time we are receding a little, and maybe we really have reached the maximum this time.

But all of this is still preliminary, for we have only 7000 stars for our analysis, so of course, we cannot be as certain as all these recent critiques of my luminosity function and determinations of star density at high galactic latitudes by Gliese, who had some 80 stars, Jones who had a hundred or so, or Murray and Sanduleak who had all of 21 stars 7 of which had no proper motion. The latter found that my star density near the North Galactic Pole must be multiplied by a factor of five, but a more recent article by Jones dealing similarly with red stars near the South Galactic Pole, comes to the conclusion that Murray's numbers must be divided by five.

Recently it has become fashionable to populate the region of the North and South Galactic Poles with very large numbers of red dwarfs, most of which have very small tangential velocities, and therefore also small proper motions. Now in 1960 I made counts on three-image color plates taken with the Palomar 48-in. Schmidt of a region near the South Galactic Pole and published the results for 4000 stars down to $m=$ $=19 \mathrm{pg}$. I found that there were far fewer red stars than expected. Now it is quite possible that I was, and am wrong in that conclusion. I applied all the tests I could think of to check my colors, galaxies, faint asteroids, white dwarfs, etc. - but even so, there is always the possibility that my data are subject to some serious but unknown systematic error. However, no one has ever bothered to analyze or discuss my data. My conclusion, you see, was then, and is now unpopular and so most people just ignore it - and this is considered modern science.

We have now finished a similar survey of proper motion stars near the North Galactic Pole, and in a year or so I expect to have the discussion available on some 10000 proper motion stars brighter than apparent magnitude $21 \mathrm{pg}$ and with motions 
larger than 0 ."18 annually in an area of $4400 \mathrm{sq}$ deg surrounding the North Galactic Pole. Again, these results will be preliminary for when we have finished the entire Palomar Proper Motion Survey we expect to have available similar data - though without colors - for half a million stars brighter than $21 \mathrm{pg}$ and with motions larger than 0".09 annually.

(2) Since I am to talk also on the space distribution and the kinematics of white dwarfs - but not on their ages, as that is outside my province - I can appropriately discuss them here, for the degenerate stars also belong to the group of low-luminosity stars. Before we start on any discussion of their properties we should first investigate how we find them, i.e. what selection effects are involved. There are three main techniques for finding white dwarfs; (1) from Proper Motions, (2) from Faint Blue Star Surveys, (3) from objective Prism Spectral Surveys. By far the most efficient is the first, and in the course of the Bruce and Palomar Proper Motion Surveys I have by now found and published more than 4500 probable, and possible white dwarfs. When an apparently faint star with a sizeable proper motion proves to be white, or perhaps we should say, much less red than expected, for its value of $H=m+5+5 \log \mu$ it is a pretty safe conclusion that it is a degenerate object. In identifying faint blue stars one does not have that certainty. I have published more than 20000 faint blue stars but I think I can say fairly definitely that most of them are not white dwarfs. The luminosities among them range all the way from $+10-$ a reasonably bright white dwarf through +3 or +4 , the typical halo Population II 'intermediate' through 0 or +1 , a typical horizontal branch star to perhaps a few blue main-sequence stars at -2 or so, to eventually even quasars at -24 or so. Extremely accurate three-color photometry might help, but is rarely conclusive; proper motions are conclusive only for stars brighter than 16-17 because a very blue white dwarf fainter than this would have only an immeasurably small proper motion, and spectra for such faint stars are just non-existent. Spectroscopic surveys could be very useful but obviously these are slow, and restricted to bright stars - I doubt whether even a dozen white dwarfs have been found this way. And here, one must point to the fact that mere spectroscopic evidence alone is obviously not reliable. At the White Dwarf Conference in St. Andrews much was made of a new very bright white dwarf in the southern hemisphere, with, apparently, little or no proper motion. If this were really a white dwarf, it should have a parallax of nearly $1^{\prime \prime}$ but nothing has been heard of it since, so I presume it has fizzled. I might also point to the number of alleged white dwarfs announced by the spectroscopists from among faint blue stars - but a very large percentage of these have evaporated again, all of these objects had relatively small proper motions which I classified as 'intermediates' with $M$ around +4 or so, instead of around +10 . The most extreme case is that of Ton 202: Greenstein now proudly claims that he was the first person ever to obtain a spectrogram of a quasar - Ton 202 - at the time he classified it as a white dwarf and thus - by his own figures underestimated the luminosity by a factor of $10^{14}$ or more, and the distance by a factor of $10^{7}$. Now the spectroscopists are very fond of saying that parallaxes estimated by proper motion people are not reliable, and are not to be compared with spectro- 
scopic determinations which are always completely accurate. I am continuously making estimates of parallax from proper motions and I have often been wrong - but never by a factor of more than ten. That one guess of Greenstein's will raise the mean error of all spectroscopic determinations of distance to a larger value than that of all proper-motion estimates for the next century.

To come back to the white dwarfs proper: we must accept the fact that the vast majority of those now known were picked up in proper motion surveys and can thus be expected to have large tangential velocities. I am therefore much puzzled by Greenstein's remark some years ago that now for the first time he had identified some Population II white dwarfs. One of the first two white dwarfs discovered $-o_{2}$ Eridani B is a high-velocity star, so are most of those found in proper motion surveys hence they must abound in Population II stars. Population I white dwarfs, with small tangential velocities may exist - even in large numbers - but as yet we have no foolproof method for finding them. Another statement of Greenstein's I take issue with is that he claims that yellow degenerates are much less frequent than previously supposed. At the St. Andrews Conference Greenstein stated that he had observed a large number of proper motion stars of yellow color but that he had found only one or two real yellow degenerates, while the vast majority proved to be $\mathrm{G}$ or $\mathrm{K}$ 'subdwarfs', i.e. high-velocity stars. Now Greenstein picked his observing list from my proper motion catalogue and chose mainly yellow stars around $m=14$ (apparent) with large proper motions, but these were mostly stars I had not designated as white dwarfs. Yellow degenerates of this color are expected to have an absolute magnitude around $M=+14$ so if one looks around apparent magnitude 14 one is searching for stars at distances of $10 \mathrm{pc}$ - and how many yellow degenerates does one expect to find. But in the Palomar Survey I have found many stars between $m=18$ and $m=20$ $(p g)$ with colors of $g$ or $k$, and large proper motions. A typical star of this kind, with $m=19 \mathrm{pg}$, color $g-k$, and a proper motion of 0.3 annually, would, if it were merely a Population II high-velocity star of $M=+9$ or brighter, have a tangential velocity of $1500 \mathrm{~km} \mathrm{~s}^{-1}$, so it is pretty certain to be a degenerate of $M=+14$ or fainter.

Summing up, it is again the same story as for the low-luminosity stars - the large majority of white dwarfs now known have been found from proper motion surveys and can therefore be expected to behave the same way, kinematically, as high-velocity stars. Again, if low-velocity white dwarfs exist we have not been able to find them in large numbers, nor do we have efficient techniques for doing so.

Whether degenerate stars of the color and temperature of a main-sequence $M$ dwarf exist we do not yet know. A few have been announced by spectroscopists, but it is fairly plain that their luminosities are not anywhere near low enough. Many years ago I pointed out that in wide binaries with one degenerate component, the main sequence star is the more luminous one, bolometrically, in an overwhelming number of cases. Hence, as I suggested about four years ago, perhaps the best prospects for M-type degenerates are the fainter components of common proper motion pairs where the primary is a yellow degenerate. We now have about a dozen of those, some with a primary of known parallax, and hence absolute magnitude 
around +13 , and of color $g$. If the secondary in such a case is five or more magnitudes fainter, and of color $m$, it would seem to be a likely prospect. From the Palomar Proper Motion Survey alone I now have about 250 such wide proper motion pairs with at least one degenerate component. I should like to emphasize again that these are the only degenerate objects for which we can really determine the masses by observing the orbital motion, but this, of course, means that we should begin now taking first epoch plates with large reflectors, and not simply wait for someone else to do this - maybe during the next century.

\section{DISCUSSION}

Buscombe: Prof. Hynek hopes to determine UBV data for stars of 16th to 20th mag. with image enhancement on the Corralitos $60 \mathrm{~cm}$ reflector near Las Cruces, New Mexico. Although initially stars in Kapteyn selected areas will be observed, he may be interested in finding-charts for a few of your tricky candidates.

Luyten: I am very glad to hear this and shall be glad to cooperate in any way I can, but I am afraid I have to add that since by now we have some 250000 new proper motions I could not begin to supply finding charts except for some very few extremely interesting stars.

Irwin: How many stars have you found with $\mu>3^{\prime \prime} \mathrm{yr}^{-1}$ for example?

Luyten: None. The machine stops searching at $\mu=2.5^{\prime \prime} \mathrm{yr}^{-1}$.

Irwin: How many $\mu>2.5^{\prime \prime} \mathrm{yr}^{-1}$ stars (on your plates) do you estimate you have missed?

Luyten: If I have to stick my neck out on this, I would say, almost zero.

Gliese: We know nothing about the real velocity dispersion of the red low-luminosity stars. We know about the differences in velocity dispersion of $\mathrm{dM}$ and $\mathrm{dMe}$ stars. Among the McCormick stars the ratio $\mathrm{dM} / \mathrm{dMe}$ is about $2: 1$. Is anything known whether this ratio varies with absolute magnitude when going to the low luminosities?

Luyten: I am afraid nothing is really known about this, since for stars fainter than $m_{p g}=15$ we have only very crude colors, and no spectra, radial velocities, or parallaxes.

Gliese: A preliminary investigation of the number of proper motion $\mathrm{M}$ stars $\left(\mu \leqslant 0\right.$ "2 $\left.\mathrm{yr}^{-1}\right)$ with $m_{p g}$ between 17 and 21 given by Prof. Luyten near the South Galactic Pole shows no evidence of a number of low-luminosity objects larger than that given by Luyten's luminosity function. In so far this count does not agree with some estimates made near the North Galactic Pole.

Cayrel de Strobel: I am very interested in your discovery of G and K stars, but I would like to know how sure you can be that these are really $\mathrm{G}$ and $\mathrm{K}$ ?

Luyten: The data were taken from the Palomar 48-in. Schmidt plates exposed with Haro's three image method. Our exposures were calibrated on SA 68 at declination $+15^{\circ}$, hence we would not expect that our stars at $-20^{\circ}$ and $-30^{\circ}$ would appear too blue. Moreover, I made three further checks - against white dwarfs, asteroids, and elliptical galaxies, and all appeared to have the right colors. Yet when getting down to 17,18 and $19 m_{p g}$ we found some red $\mathrm{M}$ stars but an unexpectedly large number of $\mathrm{F}, \mathrm{G}$ and $\mathrm{K}$ stars and I concluded therefore that these are probably halo stars. 